\title{
Droplet characterization and stability of soybean oil/palm kernel olein O/W emulsions with the presence of selected polysaccharides.
}

\begin{abstract}
Droplet characteristics, flow properties and stability of egg yolk-stabilized oil-in-water $(\mathrm{O} / \mathrm{W})$ emulsions as affected by the presence of xanthan gum (XG), carboxymethyl cellulose $(\mathrm{CMC})$, guar gum $(\mathrm{GG})$, locust bean gum (LBG) and gum Arabic (AG) were studied. The dispersed phase $(40 \%)$ of the emulsions was based on soybean oil/palm kernel olein blend (70:30) that partially crystallized during extended storage at $5{ }^{\circ} \mathrm{C}$. In freshly prepared emulsions, the presence of XG, CMC, GG and LBG had significantly decreased the droplet mean diameters. XG, LBG, GG and CMC emulsions exhibited a shear-thinning behavior but AG emulsion exhibited a Bingham plastic behavior and control (without gum) emulsion almost exhibited a Newtonian behavior. Both control and AG emulsions exhibited a severe phase separation after storage $\left(30\right.$ days, $\left.5{ }^{\circ} \mathrm{C}\right)$. The microstructure of stored $\mathrm{XG}$ emulsion showed the presence of partially coalesced droplets, explaining a large increase in its droplet mean diameters. Increases in droplet mean diameters and decreases in flow properties found for stored GG and LBG emulsions were attributed to droplet coalescence. Nevertheless, the occurrence of droplet coalescence in these emulsions was considered to be small as no free oil could be separated under centrifugation force. Increases in flow properties and excellent stability towards phase separation found for stored CMC emulsion suggested that CMC could retard partial coalescence. Thus, the results support the ability of CMC, GG and LBG in reducing partial coalescence either by providing a sufficiently thick continuous phase or by acting as a protective coating for oil droplets.
\end{abstract}

Keyword: Soybean oil; Palm kernel olein; Polysaccharides; Droplet size; Flow behavior; Emulsion stability; Partial coalescence. 